\title{
Lateral spreading inducido por licuación en Lo Rojas, Coronel, estudio de terreno
} y modelo numérico

\author{
Liquefaction-induced lateral spreading in Lo Rojas, Coronel, field study and numerical
} modelling

Fecha de entrega: 15 de diciembre 2014

Fecha de aceptación: 29 de mayo 2015

\section{Gabriel de la Maza ${ }^{1}$, Nicole Williams², Esteban Sáez ${ }^{1}, K_{\text {Kyle Rollins }}^{2}$ y Christian Ledezma ${ }^{1}$}

\footnotetext{
${ }^{1}$ Departamento de Ingeniería Estructural y Geotécnica, P. Universidad Católica de Chile, Avda. Vicuña Mackenna 4860, Macul, Santiago, Chile, gadelama@uc.cl,esaez@ing.puc.cl, ledezma@ing.puc.cl

${ }^{2}$ Department of Civil and Environmental Engineering, Brigham Young University, 368 CB, Provo, UT 84602, USA, nicoledwwilliams@gmail.com,rollinsk@byu.edu
}

El presente artículo describe un estudio de terreno detallado que se efectuó en caleta Lo Rojas, comuna de Coronel, donde se observó una importante extensión lateral gatillada por licuación durante el pasado terremoto del Maule, 2010. El estudio de terreno incluyó sondajes SPTy CPT, así como la aplicación de algunas técnicas geofisicas basadas en ondas de superficie. Sobre la base de esta información, se evaluaron expresiones empíricas de predicción de extensión lateral y se elaboró un modelo detallado de elementos finitos hidro-mecánico. Los resultados de ambos métodos se ajustan razonablemente bien a las observaciones postsísmicas en el lugar.

Palabras clave: extensión lateral, SPT, CPT, técnicas basadas en ondas de superficie, expresiones empiricas de predicción, modelación mediante elementos finitos
The paper describes a detailed field survey conducted at Lo Rojas fishermen port in Coronel, where extensive liquefaction induced lateral-spreading was reported for the 2010, Maule earthquake. The survey includes SPT and CPT soundings, as well as the use of some surface-based geophysical techniques. The data was used to evaluate empirical prediction lateral-spread expressions and to develop a detailed hydro-mechanical finite element model. Results of both empirical expressions and numerical models agree reasonably well with post-event field observations.

Keywords: lateral-spreading, SPT, CPT, surface-wave based methods, empirical prediction equations, finite element modeling

\section{Introducción}

El terremoto del 27 de febrero de 2010 causó gran daño a puertos, puentes e instalaciones industriales, afectando considerablemente las actividades económicas del país. En muchos casos, el daño está asociado al fenómeno de desplazamiento lateral inducido por licuación, en el cual grandes masas de suelo deslizan sobre suelo licuado, imponiendo enormes demandas de desplazamiento a las estructuras existentes. Caleta Lo Rojas, ubicada en Coronel a $25 \mathrm{~km}$ al sur de Concepción, es una caleta de pesca artesanal que evidenció desplazamiento lateral inducido por licuación. La estructura más dañada fue un muelle, que debió ser reemplazado luego del terremoto (Figuras 1a y 1b). Durante el estudio de terreno posterior al terremoto (Bray et al., 2012), los desplazamientos horizontales acumulados fueron medidos sumando el ancho de las grietas a lo largo de la línea indicada en la Figura 1a. El movimiento lateral máximo fue alrededor de $2.9 \mathrm{~m}$ en una distancia medida de aproximadamente $85 \mathrm{~m}$ (Figura 1c). En el presente estudio, una extensa campaña de exploración fue realizada para caracterizar el sitio, y luego se utilizaron métodos simplificados y sofisticados para reproducir los desplazamientos observados post sismo. La exploración incluyó métodos geofísicos sísmicos, y sondajes SPT y CPT. 
a)

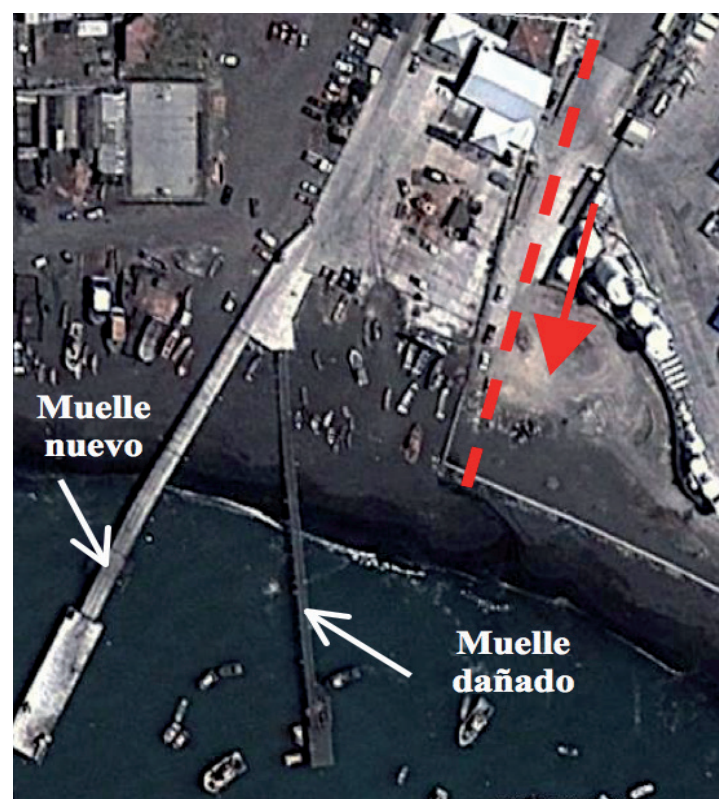

b)

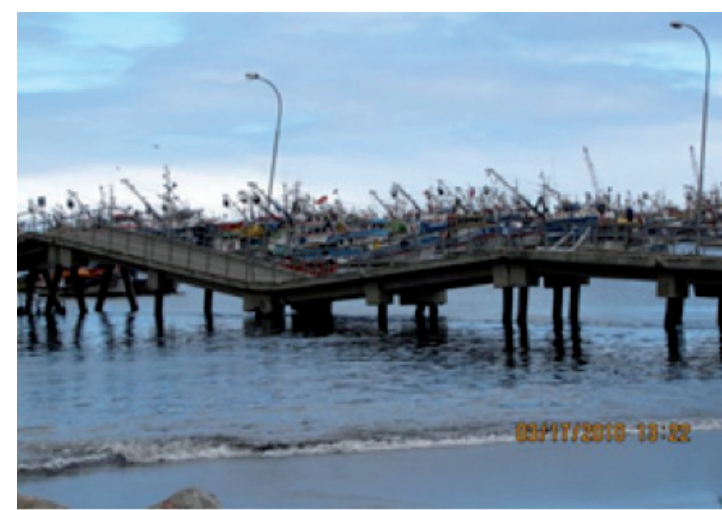

c)

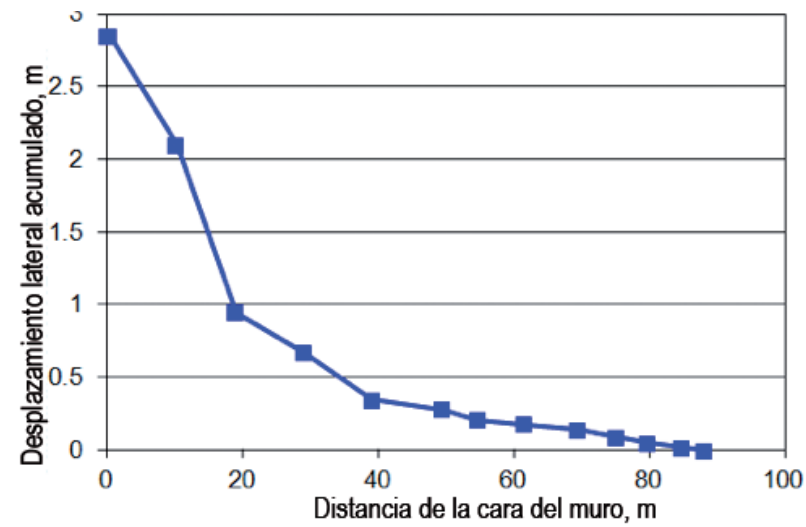

Figura 1: Caleta Lo Rojas: a) Línea de medición de desplazamiento lateral, b) muelle dañado y c) desplazamiento lateral acumulado

\section{Sondajes exploratorios}

Los sondajes SM-1 y SM-2 fueron excavados para el proyecto de construcción del nuevo muelle el 2010, al lado del muelle antiguo que fue severamente dañado durante el terremoto (Figura 1b). Cada sondaje tiene una profundidad aproximada de $25 \mathrm{~m}$ desde el nivel medio del mar. Durante esta investigación, fue ejecutado el sondaje ST-1, con una profundidad de $20 \mathrm{~m}$ desde una elevación relativa de 2.87 $\mathrm{m}$ sobre el nivel del mar. Adicionalmente, se realizaron dos Ensayos de Penetración de Cono CPT-5 y CPT-6. Las ubicaciones de los sondajes se muestran en la Figura 2.

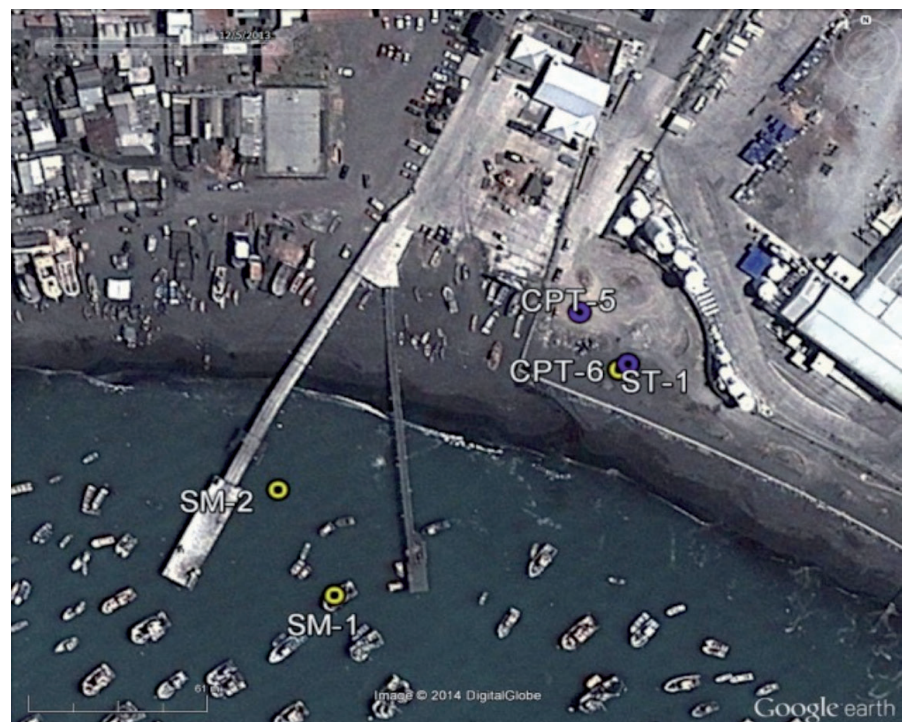

Figura 2: Ubicación de los sondajes

La Figura 3a muestra los valores del número de golpes corregido $\left(\mathrm{N}_{1}\right)_{60}$, obtenidos utilizando las correcciones sugeridas por Youd et al. (2001). Para los sondajes SM-1 y SM-2 se asume una eficiencia de energía del 60\% (la energía efectiva fue medida sólo durante la ejecución del sondaje ST-1) y un factor de corrección por confinamiento $C_{\mathrm{N}}$ (Liao y Whitman, 1986). Usando la batimetría disponible, se registró la elevación vertical absoluta respecto al nivel del mar, para cada sondaje. Los resultados muestran un gran contraste entre los primeros $20 \mathrm{~m}$ de suelo y los más profundos. En el sondaje ST-1 se puede ver una transición desde materiales sueltos a materiales densos cerca de los 14 $\mathrm{m}$ de profundidad, mientras los sondajes marinos indican que esa transición ocurre $20 \mathrm{~m}$ bajo el nivel del mar. Dado que existe una zona de fuerte pendiente en la línea de costa, en la Figura 3a solo se incluye la clasificación de suelos del sondaje ST-1, pues esta no coincide exactamente con el resto de los sondajes. El perfil geotécnico completo del sector se detalla más adelante.

La Figura 3b muestra el índice SBT (Soil Behavior Type) de los sondajes CPT, de acuerdo a Robertson (2010). Esta información indica que existen dos materiales dominantes en los primeros $20 \mathrm{~m}$ : arena y limos. Hay una transición entre estos tipos de suelos, como se observa en los valores 
a)

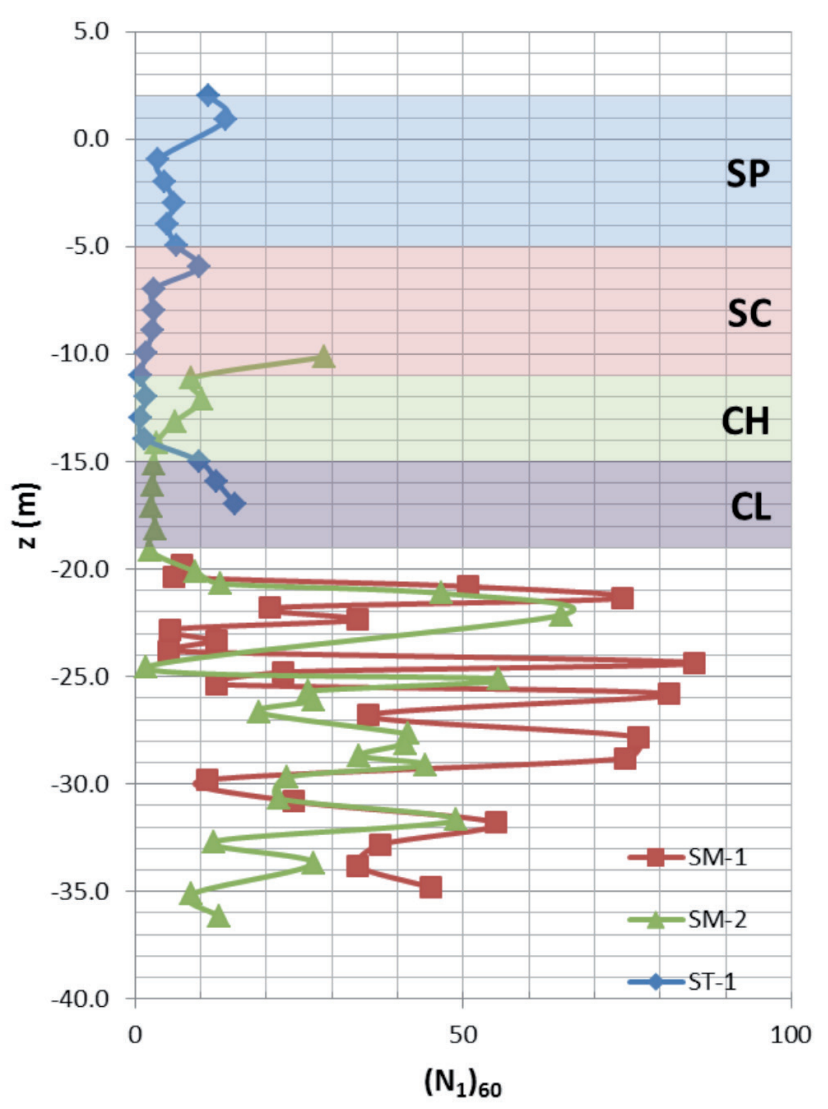

b)

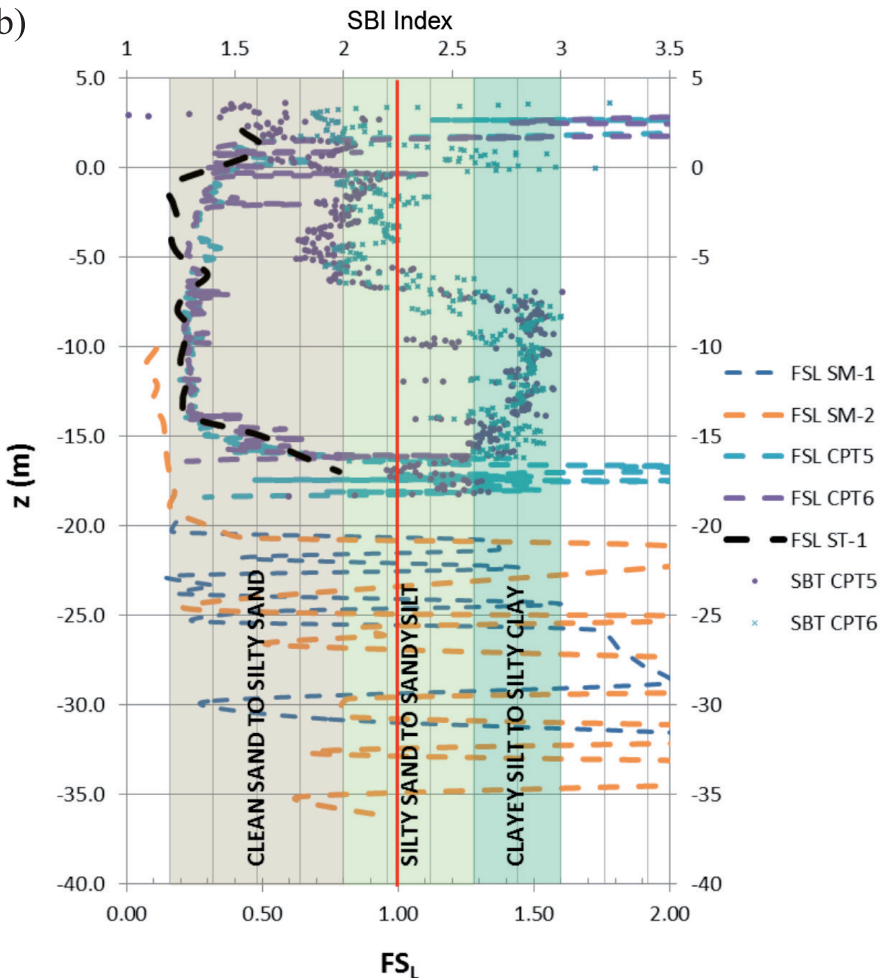

Figura 3: a) Estratigrafía y comparación de sondajes SM-1, SM-2 y ST-1, b) índice SBT del ensayo CPT y Factor de Seguridad ante Licuación en sondaje ST-1 del SBT en profundidad. En la Figura 3b, los primeros $8 \mathrm{~m}$ desde la superficie son principalmente arenas limpias que progresivamente cambian a arena limosa en los siguientes $4 \mathrm{~m}$ y finalmente se convierte en limos arcillosos a arcillas limosas a los $20 \mathrm{~m}$ de profundidad. Los valores de $\left(\mathrm{N}_{1}\right)_{60}$ para los primeros $20 \mathrm{~m}$ son prácticamente uniformes y menores a 10 golpes/pie. Además, la clasificación USCS de las muestras del sondaje ST-1 (Figura 3a) se condice con el índice SBT, mostrando clasificaciones y transiciones similares. Con valores tan bajos de $\left(\mathrm{N}_{1}\right)_{60}$ es usual que los métodos de evaluación de licuación predigan que todos los materiales licuan hasta los $20 \mathrm{~m}$ bajo el nivel del mar. El Factor de Seguridad contra la Licuación FS $_{\mathrm{L}}$ fue calculado para los ensayos SPT y CPT (Figura 3b), considerando las correcciones por contenido de finos (Youd et al., 2001). Los factores de seguridad de los ensayos CPT predicen que la capa superficial de suelo es no licuable, así como los materiales bajo la cota $z=-15 \mathrm{~m}$, lo que coincide con la información más completa del sondaje ST-1.

Bray y Sancio (2006) proponen límites que definen los suelos no licuables en términos de su Índice de Plasticidad IP. De acuerdo a estos autores, si el IP es mayor a $20 \%$ o su contenido de humedad es menor a 0.8 veces el Límite Líquido, el material no licuará. Las capas de arcilla $\mathrm{CH}$ y CL (desde $z=-11$ a $-19 \mathrm{~m}$ ) cumplen al menos una de estas condiciones, posiblemente definiendo la profundidad límite para el comportamiento licuable. Es muy probable que esta interfaz defina la superficie conductora del lateralspreading.

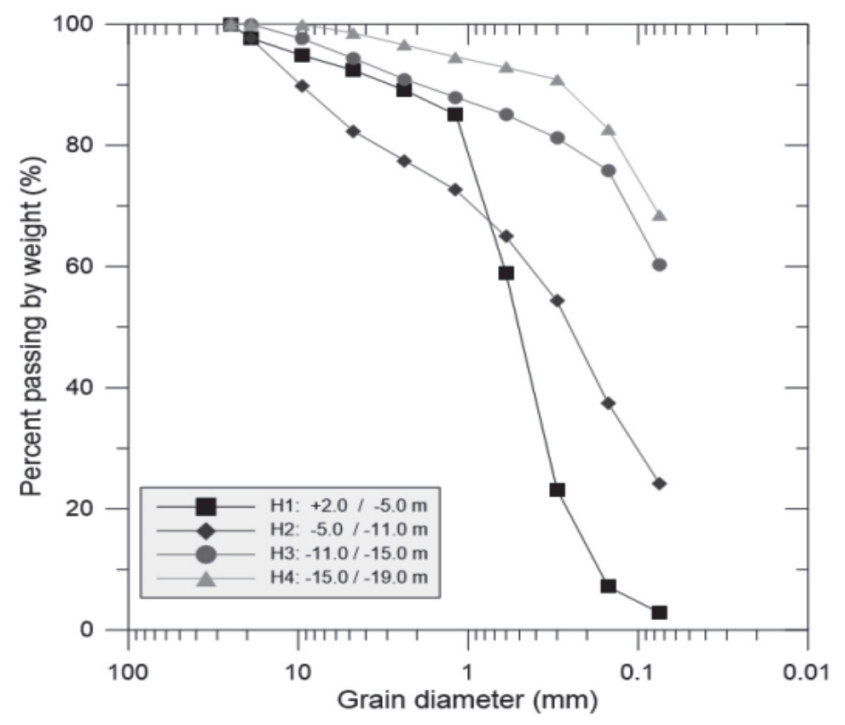

Figura 4: Curvas granulométricas promedio para cada estrato 
Las curvas granulométricas del sondaje ST-1 muestran una clara progresión de suelos granulares a suelos finos, como se muestra en la Figura 4. El contenido de finos varía progresivamente desde $1 \%$ en los primeros $6 \mathrm{~m}$, a $70 \%$ a los $15 \mathrm{~m}$ bajo el nivel del mar. Esta transición entre estratos probablemente guía el movimiento del lateral spreading inducido por licuación del estrato superficial.

\section{Exploración geofísica}

La campaña geofísica en Caleta Lo Rojas se centró en dos sectores, designados como SW-1 y SW-2. Además, la Razón Espectral Horizontal-Vertical, HSVR (Nakamura, 1989), fue calculada para 6 puntos, los cuales se muestran junto a SW-1 y SW-2 en la Figura 5. Los marcadores de colores indican la frecuencia fundamental, calculada para cada punto de acuerdo a la escala de color en el borde izquierdo (en Hertz).

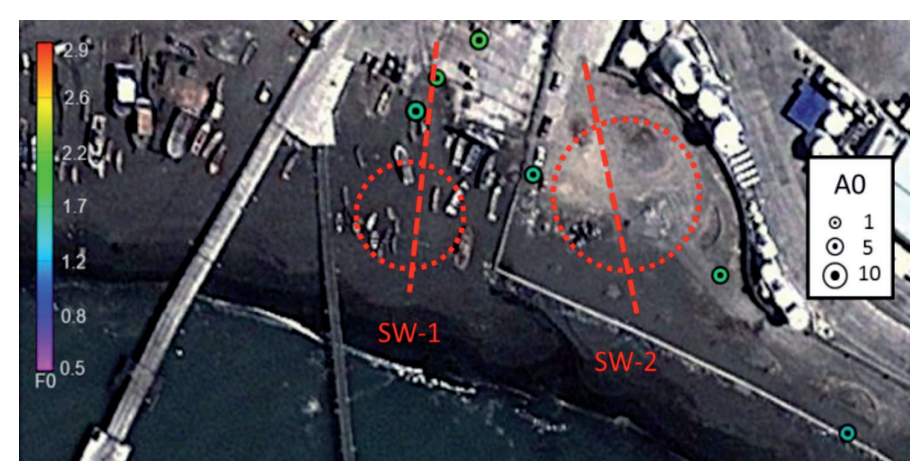

Figura 5: Exploración geofísica: resultados de Nakamura y área explorada

Los métodos geofísicos sísmicos basados en ondas superficiales caracterizan las propiedades dispersivas de un sitio basados tanto en fuentes pasivas (vibraciones ambientales) como en fuentes activas. Para esta investigación, se utilizó un sismógrafo de 24 canales con dos tipos de geófonos, de frecuencia natural de $4.5 \mathrm{~Hz}$ y $1 \mathrm{~Hz}$. Frecuencias menores implican longitudes de onda mayores, que permiten una mayor profundidad de la exploración. En este caso, debido a que las velocidades de propagación de ondas de corte son muy bajas, el uso de geófonos de $1 \mathrm{~Hz}$ fue esencial para alcanzar al menos $30 \mathrm{~m}$ de profundidad. El análisis desarrollado asume solo ondas de Rayleigh. Para experimentos de fuente controlada (ensayos activos), se utilizó el método MASW, Multichannel Analysis of Surface Waves (Park et al., 1999). Para mediciones de ruido ambiental (fuentes pasivas), se utilizó el método
SPAC (Aki, 1957) y métodos $f-k$ (Lacoss et al., 1969) para el caso de arreglos bidimensionales. Además, para el análisis de arreglos pasivos lineales, se utilizó el método Extended SPAC (ESPAC) bajo la hipótesis de campos incidentes isotrópicamente distribuidos (Hayashi, 2008).

La Figura 6a muestra las propiedades dispersivas del sector explorado SW-2, combinando los resultados de los métodos SPAC, $f-k$, ESPAC y MASW, analizados sobre un arreglo lineal y uno circular. La compatibilidad entre las diferentes técnicas es muy satisfactoria y permite una caracterización de la curva de dispersión entre 2.5 y $30 \mathrm{~Hz}$ aproximadamente. Es importante notar que la exploración geofísica no caracteriza un punto particular en el espacio, sino que representa un promedio sobre el área cubierta por los arreglos. Una vez que la curva de dispersión es obtenida, la inversión debe generar un modelo del suelo compuesto por capas horizontales con propiedades elásticas compatibles con las observaciones obtenidas en terreno, en términos de sus propiedades dispersivas (dispersión o curvas de autocorrelación). En este trabajo, se usó la extensión del Neighbourhood Algorithm NA (Sambridge, 2001) propuesto por Wathelet et al. (2008) para resolver el problema inverso. Los resultados de su aplicación a las áreas SW-1 y SW-2 se muestran en la Figura 6c. Los resultados se comparan contra mediciones directas generadas con un cono sísmico en los sondajes CPT-5 y CPT-6. Los perfiles de velocidades de ondas de corte obtenidas con la geofísica tienen una compatibilidad excelente con los perfiles obtenidos del CPT. Vale la pena mencionar que la exploración geofísica fue ejecutada varios meses antes de los sondajes CPT y SPT, con el objetivo de definir su ubicación.

Adicionalmente, la Figura $6 \mathrm{~b}$ muestra un resultado típico de HVSR (Horizontal Vertical Spectral Ratio). La gran amplitud de su peak indica la existencia de un contraste de rigidez importante entre dos suelos. La frecuencia fundamental para este sitio es $f_{0}=1.66 \mathrm{~Hz}$, con una velocidad de propagación de ondas de corte de $V_{s}=160$ $\mathrm{m} / \mathrm{s}$. Una estimación rápida de la profundidad del material más rígido $\left(V_{s}=4 H f_{0}\right)$ es $24 \mathrm{~m}$ desde la superficie del suelo, lo que es bastante consistente con la profundidad del material más denso reportada en la Figura 6c. Incluso si el material más rígido detectado con el método de Nakamura no se tratara de roca basal, este contraste de impedancias define el periodo predominante de vibración para este sitio. 
a)

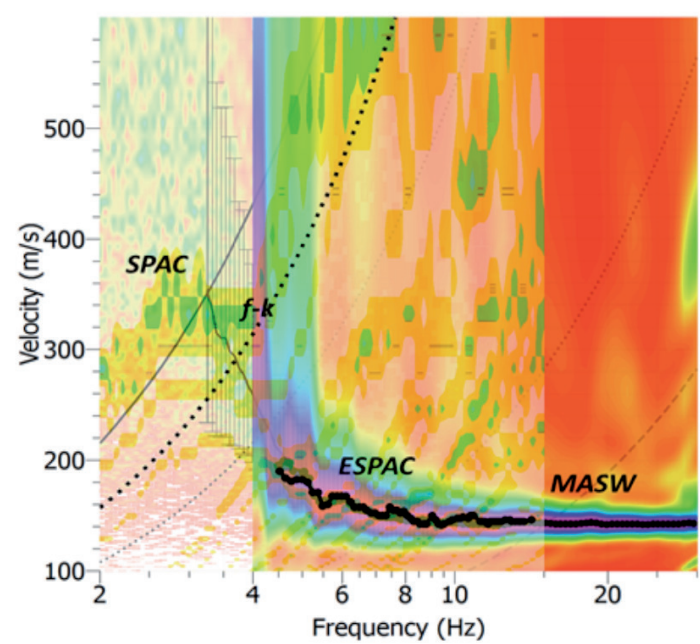

b)

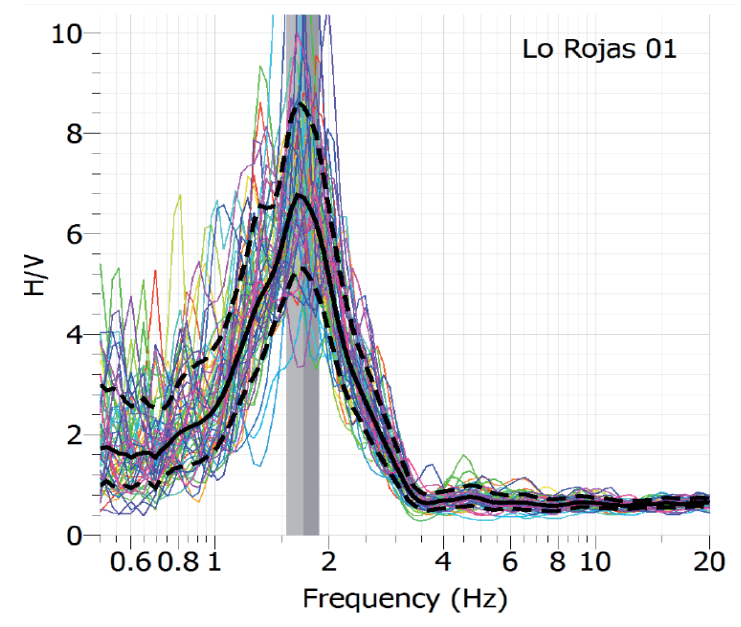

c)

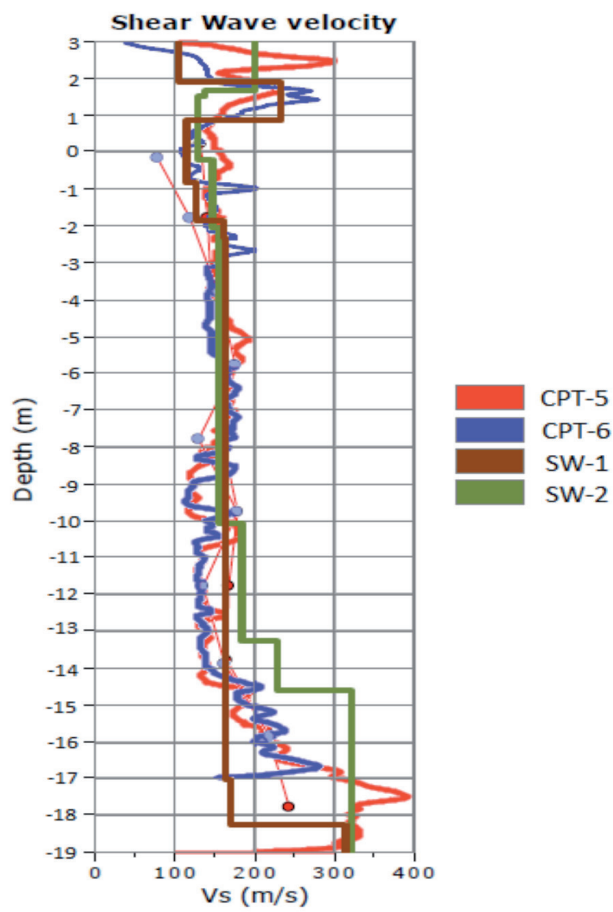

Figura 6: a) Curvas de dispersión arreglo SW-2, b) ejemplo curva de elipticidad y c) perfiles de velocidades de propagación de ondas de corte (dos sitios y comparación con CPT)

\section{Modelo geotécnico}

Para estimar el desplazamiento lateral usando tanto métodos empíricos como numéricos, se construyó un modelo geotécnico del área. Este modelo fue desarrollado basado en la batimetría disponible, provista por la Dirección de Obras Portuarias del Ministerio de Obras Públicas, a través de la sección transversal mostrada en la Figura 7a.

A pesar de que las mediciones disponibles de desplazamiento lateral no siguen exactamente la misma línea del modelo, se escogió esta sección para sacar máximo provecho a la información de batimetría. Los resultados a lo largo de esta sección no deberían ser muy diferentes de las condiciones reales del sitio. El modelo geotécnico basado en sondajes, geofísica y batimetría disponible adoptado se muestra en la Figura 7b. En esta figura, cada cuadrado tiene dimensiones de $10 \times 10 \mathrm{~m}$. La batimetría indica una pendiente abrupta con un cambio de nivel de alrededor de $10 \mathrm{~m}$ en $90 \mathrm{~m}$ de perfil, una condición que podría explicar los importantes desplazamientos laterales observados.

a)

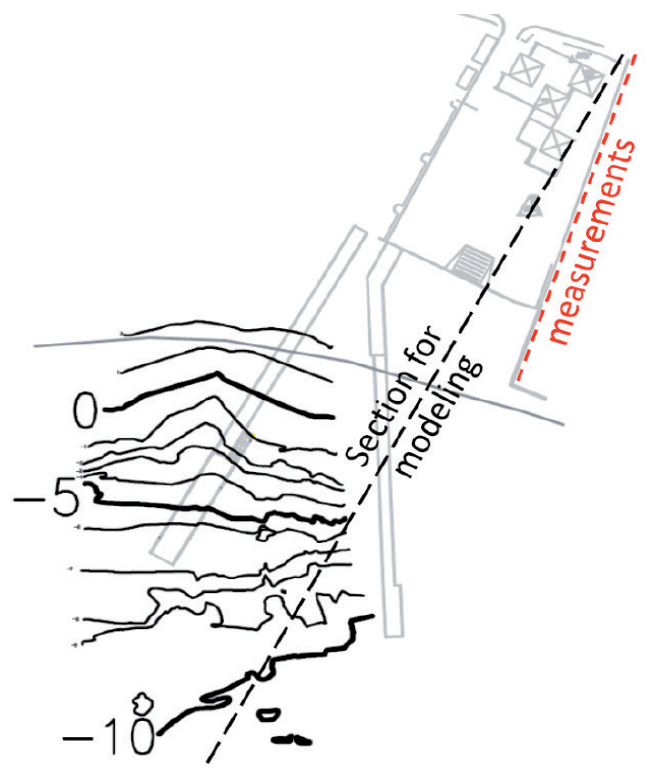

b)

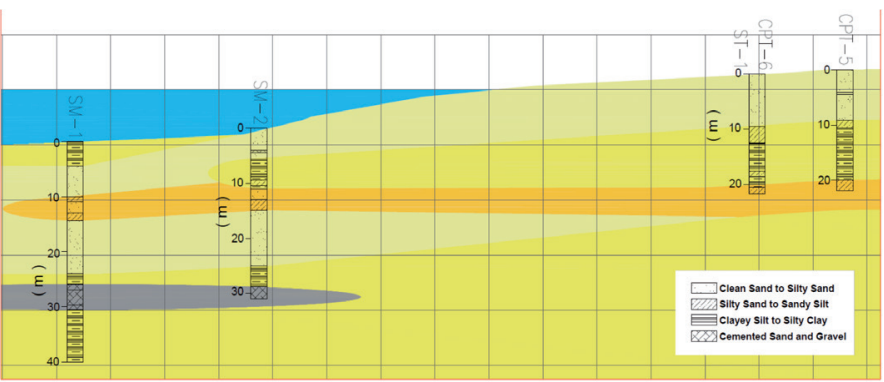

Figura 7: Modelo geotécnico, a) sección para modelar escogida para utilizar la batimetría y b) modelo geotécnico 
El perfil está caracterizado principalmente por tres materiales: H1 (arena limpia a limosa), H2 (arena limosa a limo arenoso) y H3 (limo arcilloso a arcilla limosa). Adicionalmente, un lente de arena y grava cementadas fue asumido de la información del sondaje SM-1. El material $\mathrm{H} 1$ es licuable, mientras que el material H2 es licuable probablemente solo en su porción superior (en algún punto entre $z=-11$ y $-15 \mathrm{~m}$ ) debido a su alto contenido de finos. H3 es considerado no licuable. Para el estrato de arena limpia a limosa, los sondajes marinos SM-1 y SM-2 entregan valores de $\mathrm{FS}_{\mathrm{L}}$ oscilantes bajo $z=-20 \mathrm{~m}$ (Figura 3b). Por lo tanto, el material a esta profundidad es probablemente muy denso para licuar. Para la estimación del desplazamiento lateral, presentada en las próximas dos secciones, se asume que sólo el material H1 ubicado sobre $z=-20 \mathrm{~m}$ puede licuar.

\section{Predicción empírica de lateral spreading inducido por licuefacción}

Basados en la información presentada en las secciones anteriores, se utilizaron las ecuaciones propuestas en Youd et al. (2002) para predecir los desplazamientos laterales. Esta revisión sugiere mejoras a las propuestas iniciales de Bartlett y Youd (1992, 1995). En particular, agrega una constante al término de distancia para prevenir desplazamientos irrealmente grandes y también la información disponible para el sitio. La forma general de la ecuación para condiciones de borde libre es:

$\log D_{\mathrm{H}}=-16.713+1.532 M-1.406 \log R^{*}$

- $0.012 R+0.592 \log W+0.540 \log T_{15}$

$+3.413 \log \left(100-F_{15}\right)-0.795 \log \left(D 50_{15}+0.1 \mathrm{~mm}\right)$

donde $R^{*}=R_{0}+R$, y $R_{0}=10^{(0.89 M-5.64)}, D_{\mathrm{H}}$ es el desplazamiento lateral estimado del sitio, en metros; $M$ es la magnitud de momento del terremoto; $R$ es la menor distancia horizontal desde el sitio a la fuente de la energía sísmica (en kilómetros), $R_{0}$ es una constante que es función de $M ; R^{*}$ es la distancia a la fuente sísmica modificada; $T_{15}$ es el espesor acumulado de las capas saturadas con conteo de golpes corregidos, $\left(\mathrm{N}_{1}\right)_{60}$, menor a 15 golpes/pie, en $\mathrm{m} ; F_{15}$ es el contenido de finos promedio (fracción de sedimento que pasa la malla No. 200) para los suelos en los estratos considerados en $T_{15}$, en porcentaje; $D 50_{15}$ es el tamaño de grano promedio para materiales en $T_{15}$, en mm; $W$ es la razón de borde libre definida como el alto $H$ del borde libre dividido por la distancia $L$ desde la base del borde libre para el sitio, en porcentaje.

Como se discutió en el modelo geotécnico, la capa licuable fue determinada desde la superficie hasta una elevación mínima de -11 m. De acuerdo al sondaje ST-1, el sondaje más cercano al muro donde se midieron desplazamientos, cerca de $4.5 \mathrm{~m}$ de los $11 \mathrm{~m}$ bajo el nivel del mar son arenas arcillosas con un alto IP de 11.4 y sobre $30 \%$ de finos, indicando que es poco probable que licue. Esto deja un continuo de $6.5 \mathrm{~m}$ de arena donde $\left(\mathrm{N}_{1}\right)_{60}$ es menor a 15 golpes/pie y que será utilizado para $T_{15}$, lo que es consistente con la información del CPT, donde coincide con el índice SBT bajo una elevación de $6.5 \mathrm{~m}$, como se muestra en la Figura 3b. Debido a la ausencia de muestras de los sondajes SM-1 y SM-2, el contenido de finos y tamaño de granos fueron considerados sólo a partir del sondaje ST-1. Se emplearon las mismas capas para $T_{15}, F_{15}$ $=3 \%$ y $D 50_{15}=0.44 \mathrm{~mm}$. La ubicación del borde libre fue estimada cerca de la ubicación de SM-1, donde el suelo parece nivelarse de acuerdo a la Figura $7 \mathrm{~b}$ y $W=12 \% . R$ fue considerado como la distancia del sitio a la zona de máximos levantamientos observados durante el terremoto del Maule. De acuerdo a Vargas et al. (2011), la zona de máximos levantamientos costeros se ubica en Piures, a unos $47 \mathrm{~km}$ del muelle de Lo Rojas.

Ingresando estos valores en la ecuación (1), el desplazamiento horizontal es $D_{\mathrm{H}}=10.6 \mathrm{~m}$. A pesar de la adición de la $R_{0}=155.6 \mathrm{~km}$, pareciera que la estimación de los desplazamientos es exagerada, lo que puede deberse al término de distancia relativamente pequeño. Adicionalmente, dado que la zona de máximos levantamientos puede ser difícil de estimar luego de la ocurrencia de un terremoto, se requiere de un método para estimar mejor la distancia a dicha zona. Para este estudio, se propone utilizar la mitad de la distancia desde la traza en superficie de la falla interplacas $R=80 \mathrm{~km}$. Utilizando la medida propuesta de $R$ y los mismos valores anteriores para el resto de los parámetros, se calcula con (1) que $D_{\mathrm{H}}=3.4$ $\mathrm{m}$. Se requieren estudios adicionales para sismos de gran magnitud y así definir de mejor manera el parámetro $R$.

A pesar de que el escenario de borde libre pareciera dar 
un ajuste adecuado para las mediciones post-sísmicas en Caleta Lo Rojas, la ecuación para pendientes suaves o moderadas también se examinó para complementar el estudio. Los parámetros son los mismos que para el caso de borde-libre, solo que la razón de borde-libre se omite y aparece un nuevo término $S$ que incluye la pendiente del suelo como porcentaje. Adicionalmente, los coeficientes para el escenario de pendiente moderada son diferentes, tal como se muestra a continuación:

$\log D_{\mathrm{H}}=-16.213+1.532 M-1.406 \log R^{*}-$

$0.012 R+0.338 \log S+0.540 \log T_{15}+$

$3.413 \log \left(100-F_{15}\right)-0.795 \log \left(D 50_{15}+0.1 \mathrm{~mm}\right)$

Se estima una pendiente $S=5.45 \%$ de la Figura $7 \mathrm{~b}$. Utilizando $R=40 \mathrm{~km}, D_{H}=13.9 \mathrm{~m}$, y cuando $R=80 \mathrm{~km}$, $D_{H}=4.5 \mathrm{~m}$. El valor modificado de $R$ aproxima mejor las mediciones de lateral spreading que la distancia a la zona estimada de máxima liberación de energía de las ecuaciones de Youd et al. (2002). La Tabla 1 resume los resultados de ambas ecuaciones y valores de $R$. Como la versión revisada de las ecuaciones de 2002 fue desarrollada con una base de datos que carece de un número significativo de sismos de gran magnitud, se requieren mayores estudios del término $R$ para futuras revisiones de las ecuaciones MLR.

Tabla 1: Desplazamiento horizontal $D_{\mathrm{H}}$ en $\mathrm{m}$ de Youd et al. (2002) con ecuaciones evaluadas con valores originales y modificados de $R$

\begin{tabular}{|l|c|c|}
\hline \multirow{2}{*}{$\begin{array}{l}\text { Condiciones de } \\
\text { terreno }\end{array}$} & \multicolumn{2}{|c|}{$D_{\mathrm{H}}, \mathrm{m}$} \\
\cline { 2 - 3 } & $\begin{array}{c}R \text { de Youd } \text { et al. } \\
(2002)\end{array}$ & $R$ modificado \\
\hline Cara libre & 10.6 & 3.4 \\
\hline Pendiente suave & 13.9 & 4.5 \\
\hline
\end{tabular}

\section{Modelo numérico}

Basados en la información geotécnica resumida en la Figura 7, se desarrolló un modelo de elementos finitos hidro-mecánico acoplado e inelástico para reproducir las observaciones mostradas en la Figura 1b. En este modelo, la mezcla sólido-fluido fue tratada de acuerdo a la formulación $u-p$ (Zienkiewicz y Shiomi, 1984). Esta formulación ignora la aceleración relativa del fluido y sus términos convectivos, de manera que las variables desconocidas son el desplazamiento del sólido $u$ y la presión de poros $p$. La compresibilidad de los granos del suelo se desprecia y el comportamiento del esqueleto sólido se deriva asumiendo el principio de esfuerzos efectivos propuesto por Terzaghi. Bajo estas hipótesis el problema numérico consiste en resolver la conservación del momentum de la mezcla suelo-agua, la ecuación de flujo relativo y la de conservación de la masa, simultáneamente.

La construcción de la malla de elementos finitos requiere delimitar la extensión del problema, tanto horizontal como verticalmente. Verticalmente, el modelo mostrado en la Figura 7 se extiende $5 \mathrm{~m}$ sobre la roca base y se emplearon elementos paraxiales bajo dicho nivel (Modaressi y Benzenati, 1994). La función de estos elementos es doble: por un lado se utilizan para incorporar la carga del sismo al modelo después de la deconvolución del afloramiento rocoso y, por otro lado, absorber las ondas que se originan al interior del modelo por la reflexión de las ondas en la superficie y las interfaces entre capas de suelo. La extensión horizontal del modelo se limitó a $260 \mathrm{~m}$. Esta distancia es suficientemente grande para capturar los efectos locales en la zona más escarpada de la pendiente. Dado que el modelo es completamente inelástico, los elementos absorbentes tradicionales no se pueden utilizar en los bordes verticales. Una manera de resolver este problema es utilizar bordes laterales que aseguren la condición de "campo libre" lejos de la zona de pendiente.

Como el problema no es horizontalmente periódico, una aproximación de condiciones cinemáticas periódicas estándar (Zienkiewicz et al., 1988) no es apropiada para este caso. Para imponer condiciones de campo libre en los bordes verticales se investigaron tres aproximaciones: FEM-DOF para imponer desplazamientos y presiones provenientes de una simulación de propagación unidimensional; FEMForce para imponer fuerzas laterales equivalentes de los cómputos de las columnas 1D de campo libre (Bielak et al., 2003); FEM-Column incluye en el modelo 2D columnas de campo libre y bordes laterales (Mc Gann y Arduino, 2011). Las aproximaciones FEM-DOF/Force requieren dos fases de cálculo: primero la columna de campo libre 1D debe resolverse $\mathrm{y}$, luego, los desplazamientos equivalentes o fuerzas se imponen al modelo 2D en sus bordes verticales. El acercamiento FEM-Column tiene la ventaja de requerir de un único cálculo.

La malla de elementos finitos utilizada se muestra en 
la Figura 8, donde cada color corresponde a un grupo de ítems, internamente definidos por el programa, que coinciden con las propiedades de los materiales que se han especificado. Para el método FEM-Column, la malla incluye ambas columnas de campo libre mostradas en la Figura 8. En el caso de los enfoques FEM-DOF/Force, la columna de campo libre se resuelve de manera separada y el modelo 2D incluye solo desplazamientos o fuerzas sobre los bordes verticales. La malla que incluye las columnas está compuesta por alrededor de 2800 nodos y 5300 elementos triangulares sólidos, para un total de 8000 grados de libertad (mecánicos e hidráulicos). Esta malla de elementos finitos es suficientemente fina para el problema considerado, dado que satisface la condición de tener al menos 8 nodos por longitud de onda hasta 10 Hz. Todos los cálculos se llevaron a cabo en el programa GEFDyn (Aubry y Modaressi, 1996), que es capaz de modelar problemas termo-hidro-mecánicos acoplados con condiciones estáticas y cuasi-estáticas como dinámicas. Una vez que las condiciones iniciales de esfuerzos son calculadas, el análisis sísmico se ejecuta "alrededor" de la situación estática inicial, la cual corresponde a un análisis de perturbación dinámico.

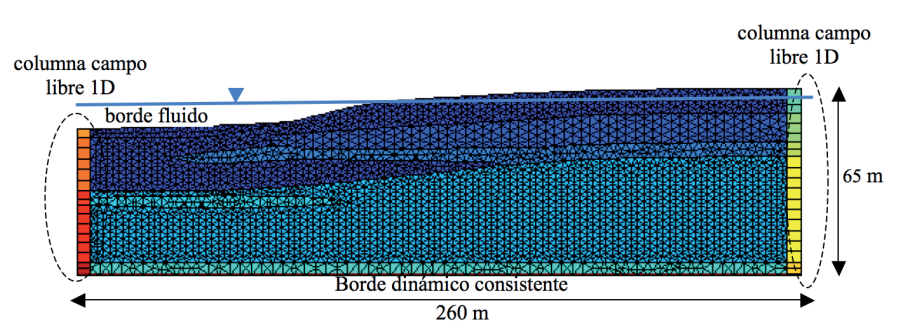

Figura 8: Modelo de elementos finitos

El modelo multi-mecanismo elasto-plástico de la Ecole Centrale Paris ECP (Hujeux, 1985) fue utilizado para representar el comportamiento de las arenas. La habilidad de este modelo de reproducir licuefacción se ha presentado en varios estudios (e.g. Lopez-Caballero y Modaressi, 2008, 2010; Sáez y Ledezma, 2014). Los parámetros para los modelos de arenas y limos, consistentes con la información de SPT y CPT del sitio, se seleccionaron de la biblioteca de materiales de los autores.

Con objeto de destacar la influencia del tratamiento de los bordes verticales en el desplazamiento lateral producido por licuación, se seleccionó la componente NS del terremoto del Maule 2010 registrado en la estación Rapel, que se encuentra sobre un afloramiento rocoso. Esta estación está ubicada aproximadamente a $500 \mathrm{~km}$ al norte de Coronel, y el registro tiene una duración de casi $90 \mathrm{~s}$ y un PGA de $0.2 \mathrm{~g}$. La Figura 9a muestra gráficos de contorno de la razón de exceso de presión de poros $R_{\mathrm{u}}$ a los $80 \mathrm{~s}$ del registro para los tres enfoques explorados. Se puede notar que licuación completa ocurre aproximadamente en los mismos sectores, independiente del enfoque adoptado. En el caso del enfoque FEM-DOF, el incremento de presión de poros parece ser más profundo comparado con las otras aproximaciones. Sólo en el caso de FEM-Force la licuación parece ser discontinua cerca de la base de la pendiente. A pesar de estas diferencias menores, los resultados son cualitativamente muy similares.
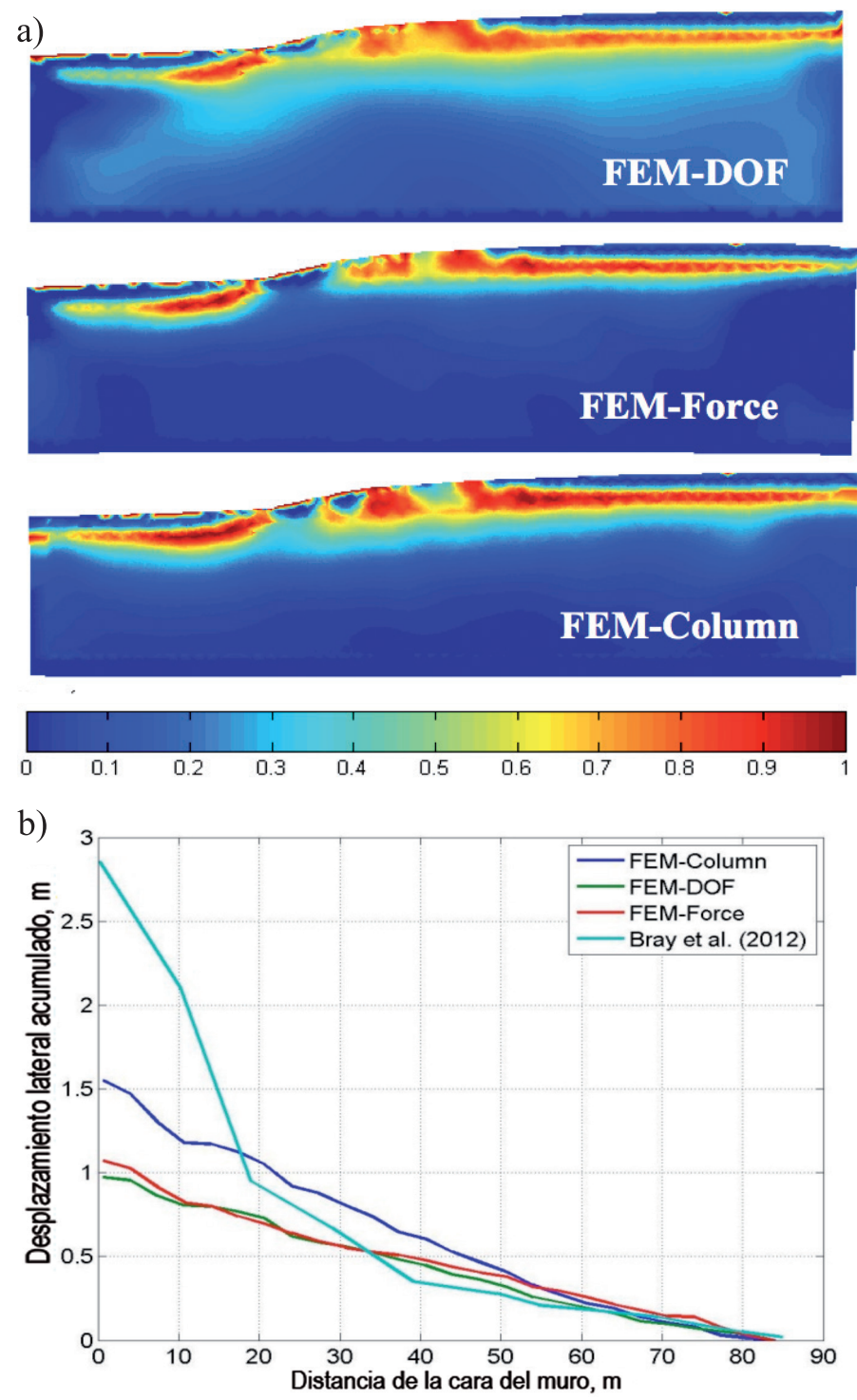

Figura 9: Comparación de condiciones de borde: a) mapas de contorno de razón de presión de poros $R_{\mathrm{u}} \mathrm{y}$ b) desplazamiento horizontal acumulado 
Para entregar comparaciones más cuantitativas, la Figura $9 \mathrm{~b}$ muestra los desplazamientos horizontales acumulados medidos en la proyección de la línea medida por Bray et al. (2012) sobre la sección considerada para el modelamiento. En términos de valores máximos, el enfoque FEM-Column entregó el valor más cercano a las mediciones de terreno (desplazamiento acumulado igual al 57\% del medido). Los resultados de FEM-DOF/Force son prácticamente idénticos. Es interesante notar que el desplazamiento acumulado entre los 20 y los $80 \mathrm{~m}$ desde la cara del muro considera como referencia durante las mediciones postsísmicas es muy similar a la obtenida por el modelo FEMColumn, pero el gran incremento en los primeros $20 \mathrm{~m}$ no es reproducido con precisión. Probablemente, grietas muy anchas fueron observadas en esa zona de la exploración en terreno, las que no pueden ser reproducidas por una estrategia de modelamiento continuo como lo es el método de los Elementos Finitos.

La estación Rapel está ubicada a 500 km de Caleta Lo Rojas, mientras que los levantamientos máximos registrados en costa se ubican a menos de $50 \mathrm{~km}$ de Coronel. Por lo tanto, el movimiento efectivo a nivel de roca base en esta área fue probablemente mayor que lo registrado en Rapel, lo que también podría explicar las diferencias observadas en los desplazamientos horizontales máximos. Desafortunadamente, el registro sobre roca más confiable y cercano para este evento es el de la estación Rapel y no hay registros similares disponibles para condiciones de suelo similares.

\section{Conclusiones}

En el presente estudio se realizó una extensa exploración que incluyó métodos geofísicos y sondajes SPT y CPT que permitieron caracterizar el sitio de Caleta Lo Rojas en la ciudad de Coronel, donde se reportaron grandes desplazamientos laterales inducidos sísmicamente para el terremoto del 27 de febrero del 2010. La información indica que el sitio se caracteriza por una pendiente escarpada de alrededor de $11 \%$ en aproximadamente $90 \mathrm{~m}$. El estrato superficial está caracterizado por $7 \mathrm{~m}$ de arenas limpias de baja densidad, seguidos por alrededor de $5 \mathrm{~m}$ de arena arcillosa. Bajo ese nivel el contenido de finos (mayor a 50\%) podría definir una frontera conductora del deslizamiento lateral. Basados en esta información, se generaron modelos simplificados y numéricos para reproducir las observaciones post-sísmicas de los desplazamientos.

Las ecuaciones de predicción empírica prueban ser muy sensibles a la distancia de la fuente sísmica. En las zonas de subducción como en Chile, el área de ruptura de los grandes terremotos pueden ser varios cientos de kilómetros. En este estudio, se propone un criterio relativo a la distancia a los mayores deslizamientos. Utilizando la distancia a la zona que concentra el 10\% de los mayores deslizamientos tectónicos, los resultados son satisfactoriamente compatibles con las mediciones in situ posteriores al terremoto.

En el modelamiento numérico, a pesar de existir varias fuentes de incertidumbre provenientes de la distribución de los materiales y sus propiedades, los resultados obtenidos por el enfoque FEM-Column son probablemente suficientemente precisos para propósitos de diseño. Un programa de ensayos de laboratorio se está actualmente desarrollando utilizando las muestras del sondaje ST-1. Éstos corresponden a ensayos monótonos triaxiales (CIU y CID) y ensayos dinámicos (triaxiales cíclicos drenados, no drenados y ensayos de columna resonante). Basados en estos resultados de laboratorio se recalibrarán los modelos constitutivos para mejorar los resultados del FEM. Además, la inclusión simultánea de las componentes horizontales y verticales de los registros sísmicos será considerada para futuras etapas de la investigación.

\section{Referencias}

Aki, K. (1957). Space and time spectra of stationary stochastic waves, with spectral reference to microtremors. Bulletin of the Earthquake Research Institute 35:415-456

Aubry, D. et Modaressi, A. (1996). GEFDyn: Manuel Scientifique. Ecole Centrale Paris (en francés).

Bartlett, S. and Youd, L. (1995). Empirical prediction of liquefaction-induced lateral spread. Journal of Geotechnical and Geoenvironmental Engineering 121(4): 316-329

Bartlett, S. and Youd, L. (1992). Empirical analysis of horizontal ground displacement generated by liquefaction-induced lateral spread. Technical Report No.NCEER-92-0021, National Center 
for Earthquake Engineering Research, Buffalo, N.Y.

Bielak, J., Loukakis, K., Hisada, Y. and Yoshimura, C. (2003). Domain reduction method for three-dimensional earthquake modeling in localized regions; Part I, Theory. Bulletin of the Seismological Society of America 93(2):817-824

Bray, J., Rollins, K., Hutchinson, T., Verdugo, R., Ledezma, C., Mylonakis, G., Assimaki, D., Montalva, G., Arduino, P., Olson, M., Kayen, R., Hashash, Y.M.A. and Candia, G. (2012). Effects of ground failure on buildings, ports, and industrial facilities. Earthquake Spectra 28, No. S1, 97-118

Bray, J. and Sancio, R. (2006). Assessment of the liquefaction susceptibility of fine-grained soils. Journal of Geotechnical and Geoenvironmental Engineering 132(9): 1165-1177

Hayashi, K. (2008). Development of the surface-wave methods and its application to site investigations. Ph.D dissertation, Kyoto University

Hujeux, J.C. (1985). Une loi de comportement pour le chargement cyclique des sols. En: Génie Parasismique, Presse ENPC, 287302 (en francés)

Lacoss, R.T., Nelly, E.J and Toksöz, M.N. (1969). Estimation of seismic noise structure using arrays. Geophysics 34(1): 21-38

Liao, S. and Whitman, R. (1986). Overburden correction factors for SPT in sand. Journal of Geotechnical Engineering 114(4): 389-411

Lopez-Caballero, F. and Modaressi, A. (2008). Numerical simulation of liquefaction effects on seismic SSI. Soil Dynamics and Earthquake Engineering 28: 85-98

Lopez-Caballero, F. and Modaressi, A. (2010). Assessment of variability and uncertainties effects on the seismic response of a liquefiable soil profile. Soil Dynamics and Earthquake Engineering 7: 600-613.

Mc Gann, C. and Arduino, P. (2011). Dynamic 2D Effective Stress Analysis of Slope. OpenSees Examples

Modaressi, H. and Benzenati I. (1994). Paraxial approximation for poroelastic media. Soil Dynamics and Earthquake Engineering 13, Issue 2, 117-129

Nakamura, Y. (1989). A method for dynamic characteristics estimation of subsurface using microtremor on the ground surface. Railway Technical Research Institute 30: 25 -30

Park, C.B., Miller, R.D. and Xia, J. (1999). Multichannel analysis of surface waves. Geophysics 64(3): 800-808

Robertson, P.K. (2010). Soil behavior type from the CPT: an update. Proceedings of $2^{\text {nd }}$ International Symposium on Cone Penetration Testing, CPT'10. Huntington Beach, CA, USA

Sáez, E. and Ledezma, C. (2014). Liquefaction mitigation using secant piles wall under a large water tank. In: Soil Liquefaction during Recent Large-Scale Earthquakes, CRC Press

Sambridge, M. (2001). Finding acceptable models in nonlinear inverse problems using a neighbourhood algorithm. Inverse Problems 17(3): 387

Vargas, G., Farías, M., Carretier, S., Tassara, A., Baize, S. and Melnick, D. (2011). Coastal uplift and tsunami effects associated to the 2010 Mw8.8 Maule earthquake in Central Chile. Andean Geology 38(1): 219-238

Wathelet, M., Jongmans, D., Ohrnberger, M. and BonnefoyClaudet, S. (2008). Array performances for ambient vibrations on a shallow structure and consequences over $\mathrm{V}_{\mathrm{s}}$ inversion. Journal of Seismology 12(1): 1-19

Youd, L., Hansen, C.M. and Bartlett, S. (2002). Revised multilinear regression equations for prediction of lateral spread displacement. Journal of Geotechnical and Geoenvironmental Engineering 128(12): 1007-1017

Youd, T.L., Idriss, I.M., Andrus, R.D., Arango, I., Castro, G., Christian, J.T., Dobry, R., Liam Finn, W.D., Harder, Jr L.F., Hynes, M.E., Ishihara, K., Koester, J.P., Liao, S., Marcuson III, W.F., Martin, G.R., Mitchell, J.K., Moriwaki, Y., Power, M.S., Robertson, P.K., Seed, R.B. and Stokoe II, K.H. (2001). Liquefaction resistance of soils: summary report from the 1996 NCEER and 1998 NCEER/NSF Workshops on Evaluation of Liquefaction Resistance of Soils. Journal of Geotechnical and Geoenvironmental Engineering 127(10): 817-833

Zienkiewicz, O., Bicanic, N. and Shen, F. (1988). Earthquake input definition and transmitting boundary conditions. In: Advances in Computational Nonlineal Mechanics, 109-138

Zienkiewicz, O. and Shiomi, T. (1984). Dynamic behaviour of saturated porous media: the generalized Biot formulation and its numerical solution. International Journal of Numerical and Analytical Methods in Geomechanics 8:71-96 